\title{
Erratum: Dihydromyricetin protects neurons in an MPTP-induced model of Parkinson's disease by suppressing glycogen synthase kinase-3 beta activity
}

Zhao-xiang REN", Ya-fei ZHAO\#, Ting CAO*, Xue-chu ZHEN*

Acta Pharmacol Sin 2016; 37 (10): 1315-24.

\# The first two authors Zhao-xiang REN and Ya-fei ZHAO contributed equally to this work.

The editors are sorry for the error. 